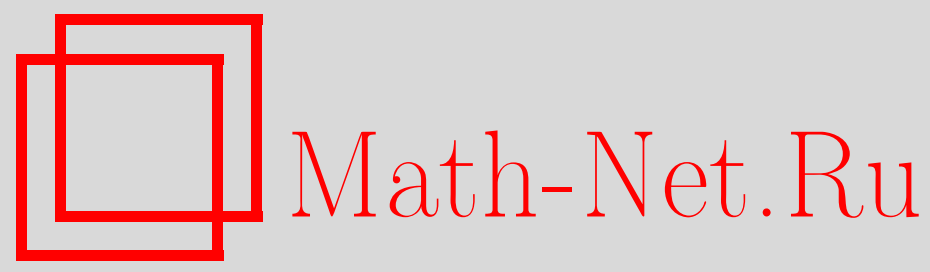

С. В. Асташкин, Ф. А. Сукочев, Сравнение сумм независимых и дизъюнктных функций в симметричных пространствах, Матем. заметки, 2004, том 76, выпуск 4, 483-489

DOI: https://doi.org/10.4213/mzm122

Использование Общероссийского математического портала Math-Net.Ru подразумевает, что вы прочитали и согласны с пользовательским соглашением http://www.mathnet.ru/rus/agreement

Параметры загрузки:

IP : 35.173 .137 .237

26 апреля 2023 г., 04:22:27

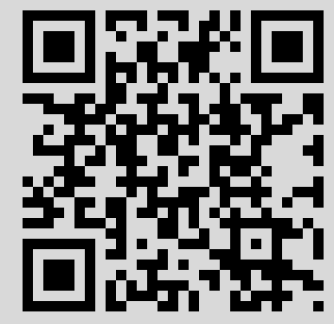




\title{
СРАВНЕНИЕ СУММ НЕЗАВИСИМЫХ И ДИЗЪЮНКТНЫХ ФУНКЦИЙ В СИММЕТРИЧНЫХ ПРОСТРАНСТВАХ
}

\author{
С. В. Асташкин, Ф. А. Сукочев
}

В работе изучаются суммы независимых функций (случайных величин) в симметричном пространстве $X$ на $[0,1]$. Наш подход является операторньм и близко связан с методами, развитыми, прежде всего, М.Ш. Браверманом. Главные результаты относятся к экспоненциальньм пространствам Орлича $\exp \left(L_{p}\right), 1 \leqslant p \leqslant \infty$, и пространствам Лоренца $\Lambda_{\psi}$. В качестве следствий мы получаем результаты, дополняюшие хорошо известную теорему Джонсона и Шехтмана о том, что из условия $L_{p} \subset X, p<\infty$, следует эквивалентность норм сумм независимых функций и их дизъюнктных "копий". Кроме того, доказано утверждение, в определенном смысле обратное к этой теореме.

Библиография: 10 названий.

1. Введение. Работа посвящена изучению сумм независимых функций в симметричных пространствах. Хорошо известно, что такие суммы ведут себя зачастую подобно суммам имеюших то же распределение дизъюнктных функций. Так, в 1970 году Розенталь [1] доказал замечательное неравенство, из которого следует, что для произвольной последовательности $\left\{f_{n}\right\}_{n=1}^{\infty}$ независимых функций из $L_{p}[0,1], p \geqslant 2$, таких, что $\int_{0}^{1} f_{k}(t) d t=0$, отображение $f_{k} \rightarrow \bar{f}_{k}$, где

$$
\bar{f}_{k}(t):=f_{k}(t-k+1) \chi_{[k-1, k)}(t), \quad t \in \mathbb{R},
$$

продолжается до изоморфизма меж ду замкнутой линейной оболочкой $\left[f_{k}\right]_{k=1}^{\infty}$ (взятой в $\left.L_{p}[0,1]\right)$ и замкнутой линейной оболочкой $\left[\bar{f}_{k}\right]_{k=1}^{\infty}$ (взятой в $L_{p}[0, \infty) \cap L_{2}[0, \infty)$ ). В $[2]$ неравенство Розенталя было обобщено на случай пространств Лоренща $L_{p, q}, 2<p<\infty$, $0<q<\infty$. Значительное продвижение в этом направлении было затем сделано Джонсоном и Шехтманом [3, теорема 1$]$, которые ввели пространства $Y_{X}$ и $Z_{X}$ на $[0, \infty)$, построенные по данному симметричному пространству $X$ на $[0,1]$, и показали, что любая конечная последовательность $\left\{f_{k}\right\}_{k=1}^{n}$ независимых, в среднем равных нулю (соответственно положительных) функций в $X$ эквивалентна (равномерно по $n$ ) последовательности их дизъюнктных сдвигов в $Y_{X}$ (соответственно $Z_{X}$ ) при условии, что $X$ содержит $L_{p}[0,1]$ при некотором $p<\infty$. В частности, из их результата непосредственно следует, что если $\left\{f_{k}\right\}_{k=1}^{\infty}$ - последовательность независимых функций в симметричном пространстве $X$ такая, что для всех $n \in \mathbb{N}$

$$
\sum_{k=1}^{n} \lambda\left(\left\{f_{k} \neq 0\right\}\right) \leqslant 1
$$

Работа поддержана Австралийским Научным Советом. 
( $\lambda$ - мера Лебега), то отображение $f_{k} \rightarrow \bar{f}_{k}, k \geqslant 1$, между $\left\{f_{k}\right\}_{k=1}^{\infty}$ и последовательностью их дизъюнктных “копий” $\left\{\bar{f}_{k}\right\}_{k=1}^{\infty}$ продолжается до изоморфизма между замкнутыми линейньми оболочками $\left[f_{k}\right]_{k=1}^{\infty}$ и $\left[\bar{f}_{k}\right]_{k=1}^{\infty}$ в $X$ при условии, что $X$ содержит $L_{p}[0,1]$ для некоторого $p<\infty$.

В этой работе изучается более общий вопрос: для каких симметричных пространств $X$ и $Y$ на [0,1] существует константа $C=C(X, Y)>0$ такая, что для каждой последовательности $\left\{f_{k}\right\}_{k=1}^{n} \subset X$ независимых функиий, удовлетворяющих условию (1), выполнено

$$
\left\|\sum_{k=1}^{n} f_{k}\right\|_{Y} \leqslant C\left\|\sum_{k=1}^{n} \bar{f}_{k}\right\|_{X}
$$

Наш подход основьвается на изучении некоторого линейного оператора и близок подходу, развитому Браверманом [4] при изучении неравенства Розенталя и его модификаций в симметричных пространствах и, в свою очередь, использующему еще более ранние идеи и вероятностные конструкции Круглова [5]. Подробнее речь об этом пойдет в п. 3 - после того, как в п. 2 будут приведены все необходимые определения. В пп. 4 и 5 будут сформулированы основные результаты работы, относящиеся к экспоненциальным пространствам Орлича $\exp \left(L_{p}\right)$ и пространствам Лоренца $\Lambda_{\psi}$. Одно из полученных при этом следствий в случае $X=Y$ представляет из себя утверждение, в определенном смысле обратное к теореме Джонсона и Шехтмана [3, теорема 1].

В п. 6, являющемся последней частью работы, мы распространим результаты на произвольные последовательности независимых функций (вообще говоря, не удовлетворяюшие условию (1)), дополнив тем самым результаты работы [3].

2. Определения и обозначения. Через $S(\Omega)(=S(\Omega, \mathscr{P}))$ будет обозначаться линейное пространство всех измеримых конечных п.в. функций на пространстве с мерой $(\Omega, \mathscr{P})$, рассматриваемое с топологией сходимости по мере на множествах конечной меры.

Банахово пространство $\left(E,\|\cdot\|_{E}\right)$ вещественнозначных функций, измеримых по Лебегу на интервале $[0, \alpha), 0<\alpha \leqslant \infty$, назьвается симметричным (или перестановочно-инвариантным), если

(i) из того, что $y \in E$ и $|x| \leqslant|y|$, следует $x \in E$ и $\|x\|_{E} \leqslant\|y\|_{E}$;

(ii) из того, что $y \in E$ и $x^{*}=y^{*}$, следует $x \in E$ и $\|x\|_{E}=\|y\|_{E}$.

Здесь и далее $\lambda$ - мера Лебега, а $x^{*}$ - невозрастающая непрерьвная справа перестановка $|x|$, т.е.

$$
x^{*}(t)=\inf \{s \geqslant 0: \lambda(\{|x|>s\}) \leqslant t\}, \quad t>0 .
$$

Если $E$ - симметричное пространство на интервале $[0, \alpha)$, то двойственное (или ассочиированное) пространство $E^{\times}$состоит из всех измеримых функций $y$, для которых

$$
\|y\|_{E^{\times}}:=\sup \left\{\int_{0}^{\alpha}|x(t) y(t)| d t: x \in E,\|x\|_{E} \leqslant 1\right\}<\infty .
$$

Если $E^{*}$ - пространство, сопряженное к $E$, то $E^{\times} \subset E^{*}$ и $E^{\times}=E^{*}$, если и только если $E$ сепарабельно. Естественное вложение $E$ в его второе двойственное $E^{\times \times}$является 
изометрической сюръекцией, если и только если $E$ максимально (или имеет свойство $\Phi a m y)$, т.е. из того, что

$$
\left\{f_{n}\right\}_{n \geqslant 1} \subseteq E, \quad f \in S[0, \alpha), \quad f_{n} \rightarrow f \text { п.в. на }[0, \alpha), \quad \sup _{n}\left\|f_{n}\right\|_{E}<\infty
$$

следует

$$
f \in E \quad \text { и } \quad\|f\|_{E} \leqslant \liminf _{n \rightarrow \infty}\left\|f_{n}\right\|_{E} .
$$

Норма $\|\cdot\|_{E}$ симметричного пространства $E$ назьвается порядково полунепрерывной, если единичньй шар $E$ замкнут в $E$ относительно сходимости почти всюду. Последнее эквивалентно тому, что естественное вложение $E$ в его второе двойственное - изометрия.

Для любого симметричного пространства $E$ на $[0, \alpha)$ справедливы непрерывные вложения

$$
L_{1}[0, \alpha) \cap L_{\infty}[0, \alpha) \subseteq E \subseteq L_{1}[0, \alpha)+L_{\infty}[0, \alpha) .
$$

Через $E^{0}$ далее будем обозначать замыкание $L_{1}[0, \alpha) \cap L_{\infty}[0, \alpha)$ в $E$. Если $E \neq L_{\infty}$, то $E^{0}$ сепарабельно. И наконец, если $E$ - симметричное пространство, то функция $\phi_{E}(t):=$ $\left\|\chi_{A}(\cdot)\right\|_{E}$, где измеримое множество $A$ удовлетворяет условию $\lambda(A)=t$, а $\chi_{A}$ - характеристическая функция этого множества, назьвается фундаментальной функиией $E$. Подробнее о симметричных пространствах см. монографии [6]- [8].

3. Свойство Круглова и оператор $\mathscr{K}$. Пусть $f$ - измеримая функция (случайная величина) на $[0,1]$ и $\mathscr{F}_{f}$ - ее функция распределения. Через $\pi(f)$ мы обозначим любую случайную величину на $[0,1]$, характеристическая функция которой задается соотношением

$$
\theta_{\pi(f)}(t)=\exp \left(\int_{-\infty}^{\infty}\left(e^{i t x}-1\right) d \mathscr{F}_{f}(x)\right) .
$$

Следующее свойство интенсивно изучалось и использовалось Браверманом [4].

ОПРЕДЕЛЕНИЕ. Говорят, что симметричное пространство $X$ имеет свойство Круглова $(X \in \mathbb{K})$, если из того, что $f \in X$, следует $\pi(f) \in X$.

Определим положительный линейньй оператор, которьй тесно связан со свойством Круглова. Пусть $\left\{E_{n}\right\}$ - последовательность попарно дизъюнктных подмножеств [0, 1],

$$
m\left(E_{n}\right)=\frac{1}{e \cdot n !}, \quad n \in \mathbb{N}
$$

Для $f \in S([0,1], \lambda)$ положим

$$
\mathscr{K} f\left(\omega_{0}, \omega_{1}, \omega_{2}, \ldots\right):=\sum_{n=1}^{\infty} \sum_{k=1}^{n} f\left(\omega_{k}\right) \chi_{E_{n}}\left(\omega_{0}\right) .
$$

Тогда

$$
\mathscr{K}: S([0,1], \lambda) \rightarrow S(\Omega, \mathscr{P}), \quad \text { где }(\Omega, \mathscr{P}):=\prod_{k=0}^{\infty}\left([0,1], \lambda_{k}\right)
$$

$\left(\lambda_{k}-\right.$ мера Лебега на $[0,1]$ для каждого $\left.k \geqslant 0\right)$. 
Оператор $\mathscr{K}$ (точнее, близкий к нему) можно определить и несколько иначе. Пусть $f \in S([0,1], \lambda)$ и $\left\{f_{n, k}\right\}_{k=1}^{n}-$ последовательность измеримых функций на $[0,1]$ такая, что

(i) функции $f_{n, 1}, f_{n, 2}, \ldots, f_{n, n}, \chi_{E_{n}}$ независимы для каждого $n \in \mathbb{N}$,

(ii) $\mathscr{F}_{f_{n, k}}=\mathscr{F}_{f}$ для всех $n \in \mathbb{N}, k=1,2, \ldots, n$.

Определим

$$
\mathscr{K}^{\prime} f(x):=\sum_{n=1}^{\infty} \sum_{k=1}^{n} f_{n, k}(x) \chi_{E_{n}}(x), \quad x \in[0,1] .
$$

Ясно, что функции распределения $\mathscr{K} f$ и $\mathscr{K}^{\prime} f$ одинаковы для любой $f \in S([0,1], \lambda)$. Так как в дальнейшем речь идет о симметричньх пространствах, то мы можем $\mathscr{K}^{\prime} f$ отождествлять с $\mathscr{K} f$.

Непосредственное вычисление, использующее (3) (или $\left(3^{\prime}\right)$ ), показывает, что $\theta_{\mathscr{K} f}(t)=\theta_{\pi(f)}(t), t \in \mathbb{R}$. Тем самым получаем простое, но важное утверждение, благодаря которому появляется возможность применять к изучению рассматриваемых вопросов интерполящионную технику.

ПРЕДЛОЖЕНИЕ 1. Если $X$ - симметричное пространство на $[0,1]$, то оператор $\mathscr{K}$ ограниченно действует в $X$ тогда и только тогда, когда $X \in \mathbb{K}$.

Следующее утверждение имеет очень важное значение для всей работы.

ТеОрема 2. Пусть $X \subseteq Y$, әде $X и Y$ - симметричные пространства на [0,1]. Рассмотрим следующие условия:

(i) существует $C>0$ такое, что (2) верно для произвольной последовательности $\left\{f_{k}\right\}_{k=1}^{n} \subset X$ независимых функиий, удовлетворяюших (1);

(ii) существует $C>0$ такое, что (2) верно для произвольной последовательности $\left\{f_{k}\right\}_{k=1}^{n} \subset X$ независимых одинаково распределенных функиий, удовлетворяющих (1);

(iii) оператор $\mathscr{K}$ ограниченно действует из $X$ в $Y^{\times \times}$;

(iii') оператор $\mathscr{K}$ ограниченно действует из $X$ в $Y$.

Справедливь импликации

$$
\left(\mathrm{iii}^{\prime}\right) \Longrightarrow(\mathrm{i}) \Longleftrightarrow \text { (ii) }
$$

Если же норма пространства $Y$ порядково полунепрерывна, то

$$
\text { (i) } \Longleftrightarrow \text { (ii) } \Longleftrightarrow \text { (iii). }
$$

4. Оператор $\mathscr{K}$ в экспоненциальных пространствах Орлича. Пусть $\Phi$ функция Орлича на $[0, \infty)$, т.е. $\Phi$ - непрерывная выпуклая возрастающая функция на $[0, \infty), \Phi(0)=0$ и $\Phi(\infty)=\infty$. Пространство Орлича $L_{\Phi}=L_{\Phi}[0,1]$ состоит из всех функций $f$, измеримых на $[0,1]$, для которых конечна норма

$$
\|f\|_{L_{\Phi}}=\inf \left\{\rho>0: \int_{0}^{1} \Phi\left(\frac{|f(t)|}{\rho}\right) d t \leqslant 1\right\} .
$$


Пространство $L_{\Phi}$ всег да максимально, а сепарабельно тогда и только тогда, когда функция $\Phi$ удовлетворяет $\Delta_{2}$-условию в $\infty$ (см. [7] или [8]).

Экспоненциальное пространство Орлича $\exp \left(L_{p}\right)$ порождается функцией $N_{p}(t):=$ $e^{t^{p}}-1$, которая при $p \geqslant 1$ вьпукла для всех $t \geqslant 0$, а при $p \in(0,1)$ выпукла для достаточно больших $t$. Кроме того, положим $\exp \left(L_{\infty}\right):=L_{\infty}$.

Из результатов [5] и [4] следует, что оператор $\mathscr{K}$ ограниченно действует в $\exp \left(L_{p}\right)$, если и только если $p \in(0,1]$. Для того чтобы охарактеризовать поведение этого оператора в пространствах $\exp \left(L_{p}\right), p \in(1, \infty]$, введем множество $\mathscr{Y}_{p}$, состоящее из всех симметричных пространств $Y$ таких, что оператор $\mathscr{K}$ ограниченно действует из $\exp \left(L_{p}\right)$ в $Y$. Определим также функции Орлича

$$
M_{p}(t):=\exp \left\{|t| \ln ^{1 / p}(e+|t|)\right\}-1, \quad t \in \mathbb{R}, \quad p>0 .
$$

Следующее утверж дение (в случае $p=\infty$ ) показывает ту важную роль, которую играет пространство $L_{M_{1}}$ в изучении систем равномерно ограниченных независимых функций (ср. [9, следствие 3.5.2]).

ТЕОРемА 3. Единственным минимальным әлементом множества $\mathscr{Y}_{p}, 1<$ $p \leqslant \infty$, упорядоченного по влохсению, является пространство Орлича $L_{M_{q}}$, где $1 / p+1 / q=1$.

СлЕДСТВИЕ 4. Пусть $p \in(1, \infty] u q=p /(p-1)$. Существует $C_{p}>0$ maкое, что для любой конечной последовательности независимых функиий $\left\{f_{k}\right\}_{k=1}^{n} \subset \exp \left(L_{p}\right)$, удовлетворяющей (1), выполнено

$$
\left\|\sum_{k=1}^{n} f_{k}\right\|_{L_{M_{q}}^{0}} \leqslant C_{p}\left\|\sum_{k=1}^{n} \bar{f}_{k}\right\|_{\exp \left(L_{p}\right)} .
$$

Более того, если некоторое симметричное пространство Y с порядково полунепрерывной нормой обладает тем свойством, что для всех последовательностей $\left\{f_{k}\right\}_{k=1}^{n} \subset \exp \left(L_{p}\right)$ таких, как выше, мы имеем $\left\|\sum_{k=1}^{n} f_{k}\right\|_{Y} \leqslant C\left\|\sum_{k=1}^{n} \bar{f}_{k}\right\|_{\exp \left(L_{p}\right)}$, mo $L_{M_{q}}^{0} \subseteq Y$.

5. Оператор $\mathscr{K}$ в пространствах Лоренца. Обозначим через $\Psi$ множество всех возрастающих вогнутых функций на $[0,1], \psi(0)=\psi(+0)=0$. Если $\psi \in \Psi$, то пространство Лоренца $\Lambda_{\psi}=\Lambda_{\psi}[0,1]$ состоит из всех функций $x(t)$, измеримых на $[0,1]$, для которых

$$
\|x\|_{\Lambda_{\psi}}:=\int_{0}^{1} x^{*}(t) d \psi(t)<\infty .
$$

ТеОрема 5. Если $\psi \in \Psi$, то оператор $\mathscr{K}$ ограничен в пространстве Лорениа $\Lambda_{\psi}$ (т.е. $\left.\Lambda_{\psi} \in \mathbb{K}\right)$ тогда и только тогда, когда существует $C>0$ такое, что

$$
\sum_{k=1}^{\infty} \psi\left(\frac{u^{k}}{k !}\right) \leqslant C \psi(u), \quad u \in(0,1] .
$$

Заметим, что условие (4) для функций $\psi \in \Psi$ возникло также в работе [10] при изучении случайных перестановок в симметричных пространствах.

Следующее утверждение в некотором смысле обратно к утверждению теоремы Джонсона и Шехтмана [3] (см. введение). 
СЛЕДСТВИЕ 6. Пусть симметричное пространство Е обладает таким свойством: для каждого максимального симметричного пространства $X \supset E$ существует $C>0$ такое, что неравенство

$$
\left\|\sum_{k=1}^{n} f_{k}\right\|_{X} \leqslant C\left\|\sum_{k=1}^{n} \bar{f}_{k}\right\|_{X}
$$

выполнено для любой последовательности $\left\{f_{k}\right\}_{k=1}^{n} \subset X$ независимых функций, удовлетворяющей (1). Тогда $E$ содержит $L_{p}[0,1]$ для некоторого $p \in[1, \infty)$.

Последнее утверждение этого пункта показьвает, что критерий ограниченности оператора $\mathscr{K}$ не может быть дан в терминах вложений.

СлЕДСТВИЕ 7. Если $\psi \in \Psi$ такова, что для каждого $\alpha \in(0,1]$ выполнено $\sup _{t \in(0,1]} t^{-\alpha} \psi(t)=\infty$, mо существует $\phi \in \Psi$ такая, что $\phi \leqslant C \psi$ при некотором $C>0$ и оператор $\mathscr{K}$ не ограничен в любом симметричном пространстве $X c$ фундаментальной функиией $\phi_{X}=\phi$.

6. Общий случай. Здесь мы рассмотрим главный вопрос (см. введение) в том случае, ког да неравенство (1), вообще говоря, не выполнено. Следуя [3], для произвольного симметричного пространства $X$ на $[0,1]$ определим функциональное пространство $Z_{X}$ на $[0, \infty)$ :

$$
Z_{X}:=\left\{f \in L_{1}[0, \infty)+L_{\infty}[0, \infty):\|f\|_{Z_{X}}^{\prime}:=\left\|f^{*} \chi_{[0,1]}\right\|_{X}+\left\|f^{*} \chi_{[1, \infty)}\right\|_{1}<\infty\right\} .
$$

Так как квазинорма $\|\cdot\|_{Z_{X}}^{\prime}$ эквивалентна норме $\|f\|_{Z_{X}}:=\left\|f^{*} \chi_{[0,1]}\right\|_{X}+\|f\|_{L_{1}(0, \infty)}$, $f \in Z_{X}$, то $Z_{X}$ является симметричным пространством на $[0, \infty)$.

Главньй результат здесь дополняет теорему Джонсона и Шехтмана [3, неравенство (4)], показьвая, что и в общей ситуации (модифицированное) неравенство (2) при условии ограниченности оператора $\mathscr{K}$ остается верным.

ТеОрема 8. Пусть $X$ и - симметричные пространства на $[0,1], X \subseteq Y$. Ecлu

(i) оператор $\mathscr{K}$ ограниченно действует из $X$ в $Y^{\times \times}$и норма $Y$ порядково полунепрерывна, или

(ii) оператор $\mathscr{K}$ ограниченно действует из $X$ в $Y$,

то существует $C>0$ такое, что для каждой последовательности $\left\{g_{i}\right\}_{i=1}^{n} \subset X$, $n \in \mathbb{N}$, независимых функций выполнено неравенство

$$
\left\|\sum_{i=1}^{n} g_{i}\right\|_{Y} \leqslant C\left\|\sum_{i=1}^{n} \bar{g}_{i}\right\|_{Z_{X}} .
$$

СЛЕДСТВИЕ 9. Пусть $X$ - интерполяционное пространство относительно банаховой пары $\left(L_{1}(0,1), L_{\infty}(0,1)\right)$. Если оператор $\mathscr{K}$ ограничен в $X$, то существует константа $C>0$ такая, что для произвольной последовательности независимых функиий $\left\{f_{k}\right\}_{k=1}^{n} \subset X, n \in \mathbb{N}$, и кажсдой последовательности

$$
\left\{g_{k}\right\}_{k=1}^{n}, \quad g_{k} \geqslant 0, \quad g_{k}^{*}=f_{k}^{*}, \quad 1 \leqslant k \leqslant n,
$$




$$
\left\|\sum_{i=1}^{n} f_{i}\right\|_{X} \leqslant C\left\|\sum_{i=1}^{n} g_{i}\right\|_{X} .
$$

В случае, когда симметричное пространство $X \supseteq L_{p}[0,1], p<\infty$, последнее утверждение было доказано в [3] (см. неравенство (10)).

\section{СПИСОК ЦИТИРОВАННОЙ ЛИТЕРАТУРЫ}

[1] Rosenthal H.P. On the subspaces of $L_{p}(p>2)$ spanned by sequences of independent random variables // Israel J. Math. 1970. V. 8. P. 273-303.

[2] Carothers N. L., Dilworth S. J. Inequalities for sums of independent random variables // Proc. Amer. Math. Soc. 1988. V. 194. P. 221-226.

[3] Johnson W. B., Schechtman G. Sums of independent random variables in rearrangement invariant function spaces // Ann. Probab. 1989. V. 17. P. 789-808.

[4] Braverman M.Sh. Independent Random Variables and Rearrangement Invariant Spaces. Cambridge: Cambridge University Press, 1994.

[5] Круглов В. М. Замечание о бесконечно делимых распределениях // Теория вероятности и ее примен. 1970. Т. 15. № 2. С. 331-336.

[6] Крейн С. Г., Петунин Ю. И., Семенов Е. М. Интерполяция линейных операторов. М.: Наука, 1978.

[7] Lindenstrauss J., Tzafriri L. Classical Banach spaces II. Function spaces. Berlin-Heidelberg-New York: Springer-Verlag, 1979.

[8] Bennett C., Sharpley R. Interpolation of Operators. New York: Academic Press, 1988.

[9] Kwapień S., Woyczyński W. A. Random Series and Stochastic Integrals: Single and Multiple: Birkhäuser, 1992.

[10] Montgomery-Smith S., Semenov E.M. Random rearrangements and operators // Amer. Math. Soc. Trans. (2). 1998. V. 184. P. 157-183.

(С. В. Асташкин) Самарский государственный университет

Поступило

(Ф.А. Сукочев) Flinders University, Australia

12.03.2004

E-mail: astashkn@ssu.samara.ru, sukochev@infoeng.flinders.edu.au 